\title{
INVESTIGACIONES
}

\section{La Evaluación Formativa de las Competencias Genéricas en la Formación del Profesorado de Educación Física*}

\author{
The Formative Evaluation of Generic Competences \\ in the Training of Physical Education Teachers
}

\author{
Antonio Fraile Aranda ${ }^{a}$, José Luis Aparicio Herguedas ${ }^{b}$, \\ Sonia Asún Dieste ${ }^{c}$, Rosario Romero Martín ${ }^{d}$ \\ a Universidad de Valladolid, Telf.: (34) 983423473 \\ Correo electrónico: afraile@mpc.uva.es \\ ${ }^{\mathrm{b}}$ Universidad de Valladolid, Telf.: (34) 983423459 \\ Correo electrónico: jlaparicio@mpc.uva.es \\ c Universidad de Zaragoza, Telf.: (34) 974238423 \\ Correo electrónico: sonasun@unizar.es \\ d Universidad de Zaragoza, Telf.: (34) 974238423 \\ Correo electrónico: rromero@unizar.es
}

\section{RESUMEN}

En este artículo se analiza el desarrollo competencial de un grupo de 58 estudiantes de $3^{\text {er }}$ curso de Educación Primaria, mención de Educación Física, en el programa de formación inicial del profesorado de la Facultad de Educación de Valladolid. Se pretende como objetivo conocer qué competencias transversales están presentes en las clases prácticas de la asignatura Expresión y Comunicación Corporal, en la que los estudiantes asumen el rol de profesor. Durante las clases se utilizan estrategias de evaluación formativa, como la coevaluación (desde observadores externos) y la autoevaluación (del propio estudiante). Para analizar los resultados obtenidos se aplicó el programa informático Atlas.ti (Versión 8.1) y el video análisis. En las conclusiones se extrae como valoración positiva la presencia de las competencias para el liderazgo, para la acción comunicativa y para la planificación y organización docente.

Palabras claves: competencias, liderazgo, comunicación, planificación docente, formación inicial del profesorado de educación física.

\section{ABSTRACT}

This article analyzes the competence development of a group of 58 students in the third year of Primary Education, Physical Education concentration in the teacher training program at the Faculty of Education, University of Valladolid. The goal of the study was to determine what transversal competences are present in practical lessons of the course Body Language and Expression, in which students assume the role of teacher. Diverse strategies of formative evaluation are used during the lessons, such as co-evaluation (by external observers) and self-appraisal (by the students themselves). To analyze the results obtained, the computer program Atlas.ti (Version 8.1) and video

Este estudio se ha llevado a cabo dentro del proyecto de I+D+i: "Las competencias docentes en la formación inicial del profesorado de educación física", convocatoria de noviembre de 2013 del Programa Estatal de Investigación, Desarrollo e Innovación Orientada a los Retos de la Sociedad, en el marco del Plan Estatal de Investigación Científica y Técnica y de Innovación 2013-2016. Referencia: EDU 2013-42024-R. Duración: 3 años (2014-2016). 
analysis were utilized. We conclude with the fact that the presence of competences in leadership, communicative action, and teacher planning and organization were valued positively.

Keywords: competences, leadership, communication, teacher planning, physical education teacher training.

\section{INTRODUCCIÓN}

En el informe publicado por la OCDE (1994) sobre la calidad en la enseñanza, para la profesión docente se confirma la necesidad de adaptarse a los nuevos desafíos y demandas de la sociedad, de las instituciones educativas y de los propios escolares, lo que requiere de la adquisición, desde la formación inicial del profesorado, de diversas competencias profesionales. Desde el Proceso de Bolonia (1999), el enfoque competencial en la enseñanza superior supone una renovación en la definición de los contenidos y métodos de enseñanza y aprendizaje, un cambio en el sistema de evaluación y una búsqueda de nuevos planteamientos didácticos acorde a este nuevo modelo (Villardón-Gallego, 2015).

En el espacio de la enseñanza superior surge como preocupación, que la formación de los estudiantes llegue más allá de la preparación científico-técnica, que facilite a los futuros docentes su incorporación al mundo laboral. Para ello, se plantea la necesidad de que estén presentes en los programas formativos, tanto las competencias docentes específicas como las de tipo transversal, como medio de adquisición de nuevos conocimientos. Se trata de capacitar al profesorado, para que pueda llegar a atender las dificultades que se vienen produciendo, en un contexto escolar sujeto cada vez más a cambios, a partir de una eficiente intervención docente (Corominas-Rovira, 2001; Villardón-Gallego, 2015; Yániz, 2007).

Este planteamiento formativo supone redefinir el proceso de enseñanza y aprendizaje, estableciendo modelos y estrategias metodológicas para la experimentación y adquisición de dichas competencias, que permitan una transferencia más real hacia el ámbito profesional (Asún, Romero \& Chivite, 2017; Salmerón, 2013). Desde esta posición, una formación basada en competencias representa la posibilidad de aprender a manejar las situaciones y los problemas complejos del ejercicio docente, siendo preciso actuar de una forma más consciente, comprometida y responsable desde ese proceso formativo (Schneckerberg \& Wildt, 2005).

En este desarrollo competencial, presente en la formación inicial del profesorado de educación física, participan todas las disciplinas que conforman su itinerario formativo. En este caso, desde la asignatura de Expresión y comunicación corporal se propone un tratamiento de los contenidos con los que abordar diferentes dimensiones relacionadas, especialmente, con las competencias interpersonales (trabajo en equipo), intrapersonales (gestión de las emociones, autoconocimiento, autoestima, resiliencia o autocontrol) e instrumentales (planificación y organización de la tarea a desarrollar, comunicación verbal y no verbal). Así, todas esas competencias se consideran esenciales para que esos futuros docentes puedan intervenir en los contextos de práctica docente, ya que les ayuda a obtener un mejor autoconocimiento personal y, a la vez, contribuyen al desarrollo de una práctica más proactiva (Fraile \& Aparicio, 2015).

Para llevar a cabo este proceso formativo, se precisa de la aplicación de un sistema de evaluación formativa, que ayude a los estudiantes a encadenar sus conocimientos y habilidades específicas, a partir de la toma de conciencia competencial, la reflexión de 
su práctica y sobre sus capacidades docentes en la acción (Asún et al., 2017). Y son las oportunidades de práctica docente, a modo de simulacros, un marco de intervención desde el que reconocer el grado de desarrollo competencial del alumnado en su desarrollo formativo como docentes. Esta propuesta didáctica parte de competencias específicas y transversales, con la finalidad de promover procesos de empoderamiento profesional en los estudiantes, debido a la conexión entre las prácticas formativas promovidas en el aula y la vinculación con su futura función docente (Maulini, Fraile \& Cano, 2015).

El valor de las prácticas formativas promovidas recae en la movilización de competencias para el diseño de estrategias de enseñanza que respondan a los desafíos diarios del aula, la utilización del conocimiento de forma aplicativa y práctica (Tejada \& Navio, 2004; Zabala \& Arnau, 2007), y al desarrollo de procesos de reflexión a partir de la acción (Schön, 1992).

Tanto el Proyecto Tuning (González \& Wagenaar, 2003) como los Libros Blancos para los títulos de Grado de Maestro de Educación Primaria, con la mención en Educación Física y de Ciencias de la Actividad Física y del Deporte, establecen competencias transversales para los perfiles docentes, que se ordenan en tres dimensiones: instrumentales, personales y sistémicas (ANECA, 2005a, 2005b).

Las competencias instrumentales recogen, entre otras, la capacidad para organizar y planificar la acción docente; lo que supone ser capaz de gestionar los objetivos y, desde ellos, los contenidos considerados relevantes para el aprendizaje del alumnado, previendo qué recursos y qué métodos son necesarios para implementar en la práctica (Monereo, 2014). También la capacidad comunicativa se desarrolla a partir de la fusión de tres estructuras comunicativas: verbal, no verbal y paralingüística puestas en contexto. Las palabras representan el universo conceptual de la realidad que se pretende transmitir, de formas paralingüísticas diversas (diferente altura tonal, intensidad sonora, oscilaciones del volumen, del timbre, cambios de ritmo, del tempo, el acento, la pronunciación) y movilizando el cuerpo a través del gesto, expresando y comunicando de forma combinada (Poyatos, 1994; Preston, 2005). Las tres estructuras están presentes en el encuentro comunicativo de forma fusionada, aunque de acuerdo con Meharabian $(1971,1972)$ la comunicación no verbal representa en el encuentro comunicativo un 55\%, la paralingüística un $38 \%$ y tan solo un $7 \%$ la comunicación verbal.

En este sentido, la comunicación no verbal requiere del despliegue de los recursos expresivos corporales, de forma que la intención de ser comprendido se apoye no solo en el valor de la palabra, sino también en el de los gestos (Learreta, Sierra \& Ruano, 2005; Ortiz, 2002). Para Knapp (1992) la comunicación no verbal resulta de la integración del comportamiento cinésico o movimiento corporal, que está integrado a su vez por los gestos y los movimientos expresivos corporales de extremidades, cabeza, expresiones faciales, conducta de los ojos o la propia postura corporal; también de la conducta táctil, el paralenguaje, la proxémica o uso del espacio social o personal, y las propias características físicas de la persona. Todos los elementos desplegados en el hecho comunicativo permiten llegar más allá del propio potencial comunicador de la palabra, complementándola, enfatizándola, acentuándola o sustituyéndola (Ekman \& Friesen, 1969).

Las competencias personales integran diversas capacidades vinculadas a la inteligencia social, que requieren del conocimiento y funcionamiento de las relaciones sociales y de un comportamiento inteligente (Goleman, 2006). De tal ejercicio dependen competencias docentes como la capacidad crítica y autocrítica, la cooperación y el trabajo en equipo, el 
compromiso ético y las habilidades en las relaciones interpersonales, a partir de la vivencia emocional que se experimenta en la interacción social y que nos permite reaccionar y relacionarnos mejor (Ruano, 2013). En un estudio sobre competencias interpersonales en la asignatura de expresión corporal se pudo observar cómo capacidades como la empatía o la escucha activa constituían una parte importante de las habilidades interpersonales presentes en el aula en contextos de simulación docente (Aparicio \& Fraile, 2016); además, tales capacidades son facilitadoras de las relaciones interpersonales de compañerismo, desde la preocupación por los demás, desde la amistad y, en definitiva, desde la vivencia, la expresión y la comunicación de las emociones que están inmersas en estos procesos relacionales, siendo necesario regular el impacto tanto en el plano personal como en su proyección hacia los demás (Ruano, 2013).

Por último, el desarrollo de las competencias sistémicas requiere del manejo de las habilidades relacionadas con los sistemas como totalidad, que exigen de un saber comprender, conocer y sensibilizarse de forma combinada, para conseguir vislumbrar la manera de relacionarse y agruparse las partes de un todo (González \& Wagenaar, 2003). En este grupo estarían contenidas las competencias adaptativas, de aprendizaje permanente y de liderazgo.

El liderazgo en el ámbito educativo surge a partir de ciertos rasgos de la persona que resultan eficaces según el contexto, tanto a nivel interno como para liderar a los miembros grupos en el marco de un proyecto compartido. Esta conceptualización integra, por tanto, al propio docente, a su grupo de referencia, al contexto socioeducativo y al proyecto compartido (Lorenzo, 2005). Una de las cualidades extendidas del liderazgo es la de dinamización y movilización grupal: los líderes actúan a favor de la consecución de un proyecto común, siendo el autoconocimiento, la motivación, la dinamización grupal, la capacidad para despertar entusiasmo y generar, con todo ello, un clima positivo de aula en donde están presente las emociones (Goleman, Boyatzis \& McKee, 2002; Martens, 1987).

Con el presente trabajo se pretende conocer y analizar qué competencias transversales están presentes en las prácticas docentes de los estudiantes durante el desarrollo de la asignatura Expresión y comunicación corporal.

\section{MÉTODO}

Esta experiencia se desarrolló durante el curso 2015/2016 en la asignatura Expresión y comunicación corporal, en la que participaron estudiantes de educación física de $3^{\text {er }}$ curso de la Facultad de Educación de la Universidad de Valladolid. Se contó con una muestra de 58 estudiantes entre 20 y 26 años.

Se parte de un procedimiento metodológico (Figura 1) desde el que se impulsa la práctica docente de los estudiantes en forma de simulacros, donde todos asumen el rol de "docentes", a partir de los contenidos didácticos de la asignatura de expresión corporal, debiendo diseñar, desarrollar y autoevaluar su intervención. De la misma forma, también todos asumen el rol de "observadores y coevaluadores", quienes en grupos de seis estudiantes por cada compañero "docente", coevalúan apoyados en una rúbrica de observación, informando y evaluando a los compañeros "docentes”, con carácter inmediato, sobre aquellas competencias y capacidades que han considerado mejor desarrolladas, completando para ello y por escrito un informe competencial. 
Figura 1. Estrategia didáctica para la experiencia de innovación docente

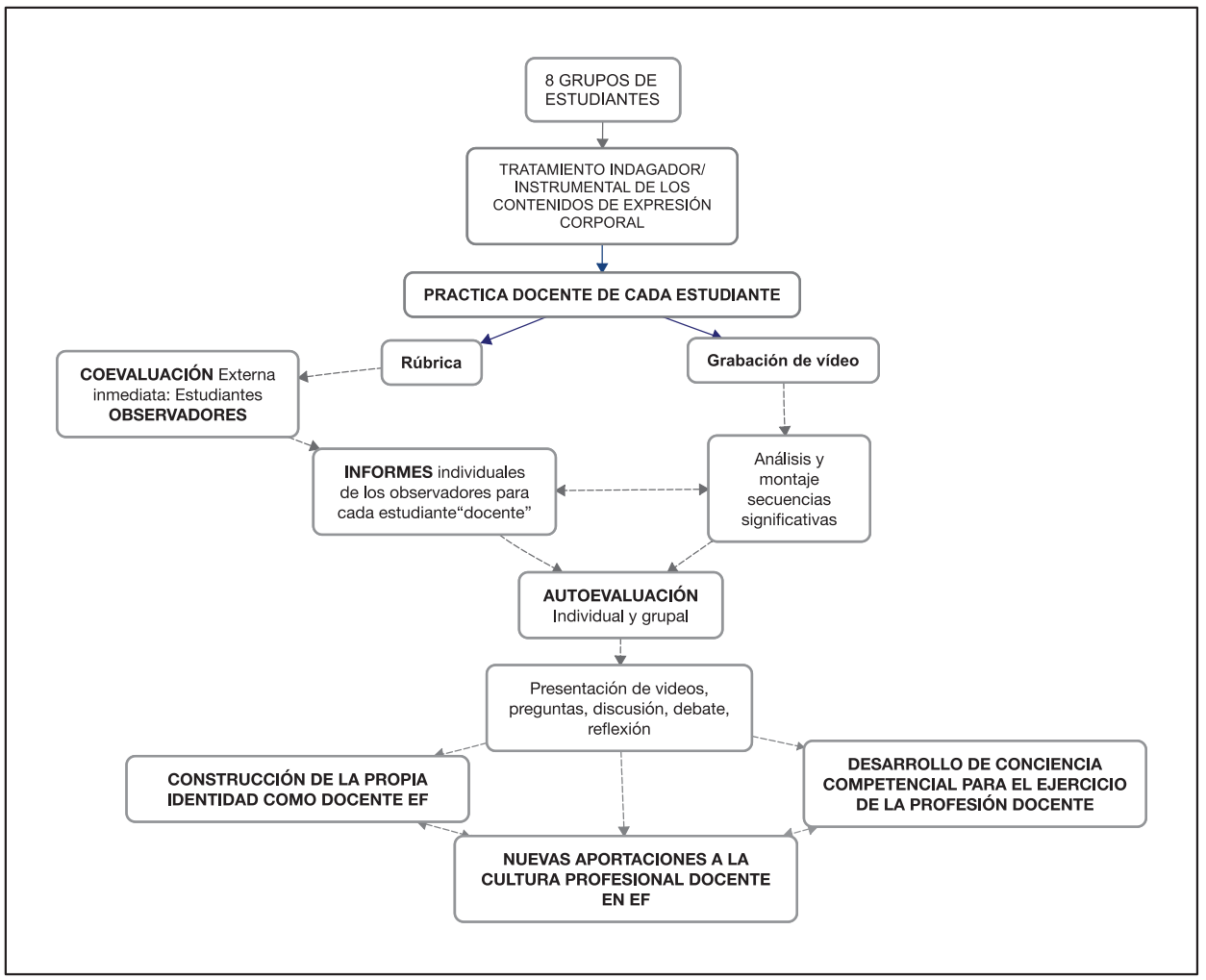

Los simulacros docentes son propuestas didácticas de intervención que planifican y dinamizan los estudiantes que asumen el rol de "docentes". Cada uno de ellos tiene asignada una dimensión corporal expresiva que estructura la materia (Lúdico-hedonista, Espacial, Rítmica, Comunicativa, Emocional, Cooperativa, Introyectiva y Creativa) y de la que se especializan. Tras el estudio pormenorizado de la dimensión asignada y la elaboración de un informe final al respecto, trabajan en la aplicación práctica de lo estudiado, proponiendo diversas actividades de enseñanza y aprendizaje a sus propios compañeros. Esta aplicabilidad se cristaliza en el ejercicio de la docencia por parte de cada uno de los miembros del grupo, poniendo en práctica las acciones didácticas planificadas durante un tiempo máximo de diez minutos, no siendo más de noventa la actuación general del grupo.

Las actividades previstas para su propuesta contienen una actividad expresiva corporal por cada estudiante "docente" que ejerce, junto con las variantes que considere oportunas a fin de diversificar la propuesta expresiva.

En la rúbrica de observación diseñada a partir de la propuesta de Amado, SánchezMiguel, Leo, Sánchez-Oliva y García-Calvo (2012), se establecen cinco competencias a observar (planificación y organización docente, comunicativa, liderazgo y resolución de conflictos, trabajo en equipo y emocional), y cuatro grados de desarrollo que ubican la 
Estudios Pedagógicos XLIV, ํㅜㄹ 2: 39-53, 2018

LA EVALUACIÓN FORMATIVA DE LAS COMPETENCIAS GENÉRICAS EN LA FORMACIÓN DEL PROFESORADO DE EDUCACIÓN FÍSICA

intervención docente en los niveles de "excelente", "admirable", "mejorable" y "amateur". Algunos de los ítems propuestos para cada competencia se recogen en la Tabla 1.

Tabla 1. Estrategia didáctica para la experiencia de innovación docente

\begin{tabular}{|l|l|}
\hline Competencia & Ítem de Observación \\
\hline $\begin{array}{l}\text { Planificación y } \\
\text { organización docente }\end{array}$ & $\begin{array}{l}\text { Cumple el plan de clase (objetivos, contenidos, metodología } \\
\text { prevista, recursos y materiales, criterios de evaluación) }\end{array}$ \\
\hline Comunicativa & Comunica de forma clara y concisa \\
\hline Liderazgo & Gestiona la clase y genera buen clima \\
\hline Trabajo en equipo & $\begin{array}{l}\text { He aceptado y cumplido las normas de funcionamiento del } \\
\text { grupo, motivando, mostrado respeto y apoyo hacia sus miembros }\end{array}$ \\
\hline Emocional & Muestra seguridad y confianza en uno mismo \\
\hline
\end{tabular}

Fuente. Elaboración propia

Para completar el procedimiento metodológico se registra mediante grabación en video cada intervención docente, con la finalidad de evidenciar la información procedente de la coevaluación y ayudar, con ello, a reforzar el ejercicio de autoevaluación de los "docentes".

Se parte del análisis documental de los 58 informes de los "coevaluadores" haciendo uso del software Atlas.ti (v. 8.1) y estableciendo un proceso de codificación para cada uno de ellos, siendo estos los documentos primarios del proceso analítico. Las citas suponen cada uno de los fragmentos de texto, que por su significación fueron asociados a un código. Los códigos sirvieron para ordenar y categorizar dichos fragmentos de forma que fuera posible, tras este proceso, establecer un escenario para el análisis y las conclusiones posteriores, a partir de un enfoque básico de codificación de carácter descriptivo que utiliza sustantivos y condensa, en ellos, el significado del pasaje textual seleccionado (Miles, Huberman \& Saldaña, 2014).

Para abordar los aspectos de credibilidad de la investigación, siguiendo a Guba (1985), y a fin de conservar el valor holístico de las relaciones que configuran el fenómeno objeto de investigación, se estableció durante el proceso investigativo un trabajo prolongado en el tiempo y en un mismo lugar (aula clase), haciendo uso de un mismo planteamiento metodológico, con el objeto de acostumbrar a los participantes a la presencia de investigadores y observadores y a una misma dinámica de actuación. De la misma forma se favoreció la observación persistente de los participantes e investigadores, a fin de identificar las cualidades perseverantes o características atípicas del fenómeno objeto de investigación.

Además, tanto durante como después del proceso, se pusieron en contraste los avances en el cocimiento del fenómeno investigado con otros investigadores, desarrollando dinámicas de juicio crítico; también se realizó un proceso de triangulación desde varias de fuentes de datos, siendo el análisis documental y el videoanálisis los desarrollados para contrastar los descubrimientos e interpretaciones. Se estableció una coherencia estructural a la hora de analizar los datos provenientes de las diferentes fuentes, y se pusieron en contraste a fin interpretar las contradicciones en relación con las características y cualidades de un mismo 
dato, mitigando los efectos de los conflictos internos en la interpretación de los mismos para garantizar consistencia, sincronización y lógica en la interpretación. Finalmente, se facilitó tras la aplicación de las estrategias de autoevaluación y coevaluación, un proceso de comprobación a modo de discusión con los participantes, acerca de los datos que se fueron obteniendo. Tal proceso se obtuvo a través de grabaciones audio que recogen el resultado de este feedback por parte de los participantes en el estudio.

Por último, el valor didáctico de esta experiencia formativa abre para los estudiantes la posibilidad de asumir no solo un rol de "docentes" (que programa, aplica y evalúa una intervención docente), sino también vivenciar su papel de "observadores y coevaluadores", a partir de la visualización, recolección y valoración de la intervención de sus propios compañeros. Esto representa un ejemplo de cómo desarrollar la competencia colaborativa para avanzar en su futuro ejercicio profesional docente y construir su propia identidad como educadores físicos, a partir de las experiencias compartidas (Velázquez, Fraile, \& López-Pastor, 2014).

\section{RESULTADOS}

Con relación a la cuestión objeto de investigación: conocer qué competencias transversales están presente en las prácticas docentes de los estudiantes durante el desarrollo de la asignatura Expresión y comunicación corporal, subyacen con fuerza (Figura 2) la competencia para el liderazgo (36 veces codificado), la competencia comunicativa (frecuencia de 33 códigos), y la competencia para la planificación y organización de las tareas docentes (17 veces codificado), por ello se dedica este apartado a tales competencias.

Figura 2. Estudio de las frecuencias de los códigos

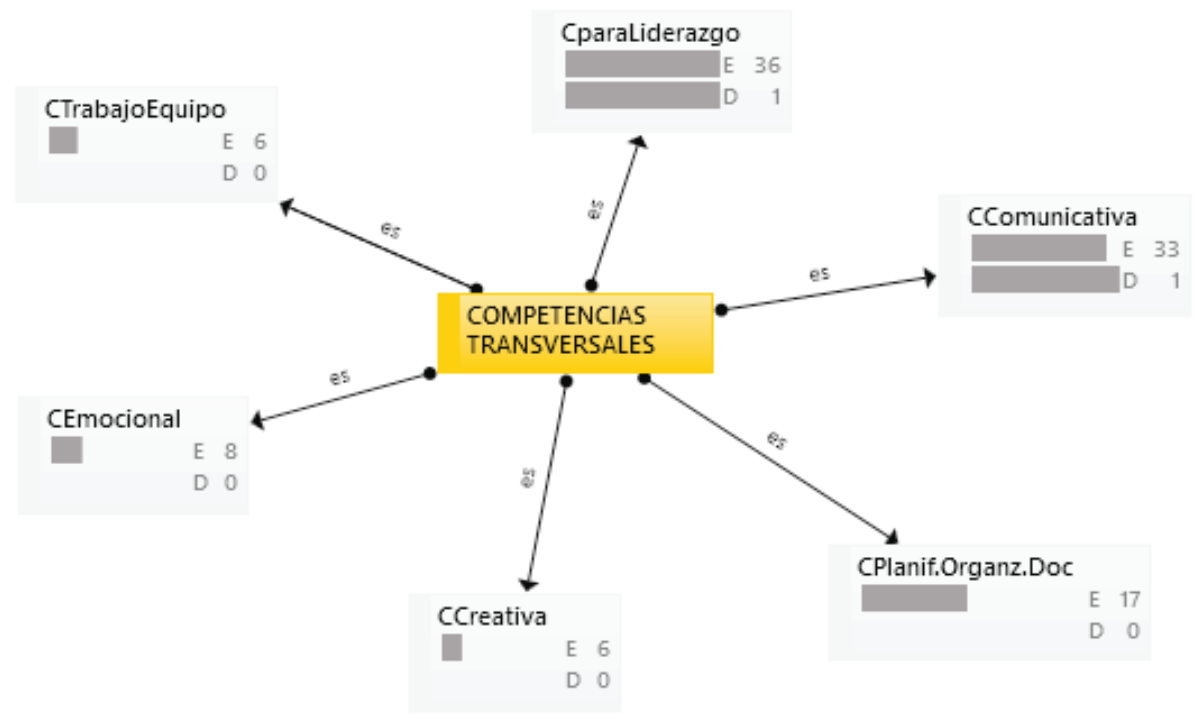


La forma de ejercer un modelo de liderazgo educativo que se identifica, para los coevaluadores, con la capacidad de motivación y de dinamización grupal y con la capacidad de animar constantemente al estudiante en el proceso de aprendizaje, se hace patente en el pensamiento del estudiante observador desde las siguientes evidencias: "capta muy bien la atención de todos y les motiva dirigiéndose a ellos" (1:64); "lleva a los alumnos a dónde él quiere y cómo quiere, motivando y dinamizando activamente" (1:10). Igualmente, se aprecian aspectos relativos a un tipo de liderazgo prosocial, preocupado por el ambiente grupal, en la ayuda mutua y la comunicación, con el fin de sacar lo mejor de cada uno. Este aspecto lo expresan en "fomenta el buen clima de aula" (1:60); "es generador de buen ambiente" (1:68); "ayuda a todos los grupos, con buena coordinación y clima de aula" (1:65); "pasa por todos los grupos para ayudar" (1:89).

En segundo lugar, saber comunicarse, desde el punto de vista verbal, se relaciona en aspectos del manejo de la voz en cuanto a intensidad, volumen y proyección, con el fin de garantizar la llegada con claridad del mensaje didáctico. De la misma manera, se observa la ejemplificación como herramienta de apoyo al mensaje transmitido: "explicación clara... se entendía bien" (1:4); "adecuada comunicación, manejo de las instrucciones y del grupo, eficaz en lo que quiere transmitir" (1:8); "buena explicación: activa, voz clara"; "buena explicación, concisa y tono adecuado, pone ejemplos" (1:66).

Además, se aprecia la relación integrada que se establece en el hecho comunicativo entre el manejo del lenguaje verbal y del lenguaje corporal, de forma que cuerpo y movimiento, integrados junto con la palabra en su intervención docente, facilitan la transmisión de la información. En este sentido, se reconoce así el uso y manejo del espacio para contextualizar la distancia conversacional, en un hecho comunicativo que se aprovecha de la simbología del gesto para la transmisión de los mensajes didácticos. Estos aspectos se aprecian en evidencias como "una buena comunicación y una actitud motivadora con tono de voz e implicación con palabras y gestos" (1:14), y "explicación clara, sencilla, eficaz, con buena gestualidad y desplazándose por el espacio" (1:21).

También, la transmisión del mensaje didáctico requiere de esfuerzos didácticos para asegurar la retroalimentación y el feedback; es decir, para verificar que dicho mensaje se graba como información aprendida. En este sentido, se reconocen en la intervención de los compañeros "docentes" algunos rasgos de esta característica: "buen comunicador, realiza feedback" (1:36); "pasaba por todos los grupos, corregía" (1:44); "resuelve las dudas de manera clara, realiza feedback durante la actividad y buena explicación en general" (1:50).

La competencia planificar y organizar la acción docente parece relacionarse con la capacidad para adecuar la intencionalidad establecida sobre los logros alcanzados. Esto es, si cada uno de los componentes previstos en las propuestas de intervención de los "docentes" (objetivos, contenidos, metodología, sistema de evaluación) son finalmente desarrollados conforme lo establecido en la ficha entregada por los "docentes", antes de su práctica. Este aspecto se evidencia en "cumple objetivos y contenidos expuestos al principio" (1:25); "actividad que consigue el objetivo" (1:48); "se cumplen los objetivos establecidos" (1:49).

De la misma manera, se observa la necesidad de facilitar la práctica de los participantes, asegurando que los niveles de complejidad y dificultad de ejecución de las tareas planteadas por los "docentes" son asumibles por todos, lo cual se puede establecer añadiendo progresividad en los niveles de dificultad o introduciendo en sus propuestas, variantes novedosas, motivadoras y facilitadoras de la ejecución. Esta dimensión se observa 
en evidencias como "buen planteamiento de la actividad y de introducción de variantes" (1:37); "la actividad introductoria es animada y motivante; la selección de la actividad es acertada para ese momento de la sesión" (1:32); "actividad novedosa y el aumento de la dificultad es progresiva" (1:39); "buena variante y música adecuada" (1:45).

También se aprecia la buena elección de los estilos de enseñanza, valorando cuál de ellos se adecúa de manera más coherente a la actividad de aprendizaje y al objetivo a alcanzar. Por ello, al reconocer en la práctica de sus compañeros "docentes" estilos de carácter más directivos, se valoran sobre la necesidad de su uso en el contexto de aprendizaje, como en el caso de tener que enseñar aspectos técnicos o de forma analítica algunos gestos o movimientos relativos al baile y la danza. Esto se manifiesta en "se es fiel al mando directo para enseñar algunos pasos de baile y estaría justificado" (1:34).

Finalmente, se realiza un análisis de co-ocurrencia de códigos (code cooccurrence table) (Tabla 2), para conocer la frecuencia de aparición de una pareja de códigos en un mismo segmento de los documentos primarios, examinar las relaciones entre los códigos y averiguar de qué forma se emparejan (Friese, 2012). En este sentido, observamos una relación entre la competencia comunicativa y la competencia para el liderazgo. Su vinculación estima datos positivos con una frecuencia de 14 y un índice de 0,26.

Tabla 2. Co-ocurrencia de códigos

\begin{tabular}{|l|c|c|c|c|c|c|}
\hline \multicolumn{7}{|c|}{ Co-ocurrencia de códigos } \\
\hline & CComunicativa & CCreativa & CEmocional & CLiderazgo & CPlanificación & CTrabajoEquipo \\
\hline CComunicativa & & & $3(0,08)$ & $14(0,26)$ & & \\
\hline CCreativa & & & & $1(0,02)$ & $1(0,05)$ & $1(0,09)$ \\
\hline CEmocional & $3(0,08)$ & & & $5(0,13)$ & & $1(0,08)$ \\
\hline CLiderazgo & $14(0,26)$ & $1(0,02)$ & $5(0,13)$ & & & $2(0,05)$ \\
\hline CPlanificación & & $1(0,05)$ & & & & \\
\hline CTrabajoEquipo & & $1(0,09)$ & $1(0,08)$ & $1(0.05)$ & & \\
\hline
\end{tabular}

A partir de esta relación, se aprecia que la actitud motivadora y orientadora que caracteriza al líder educativo requiere del despliegue de recursos comunicativos verbales y gestuales que permitan transmitir y sostener en el tiempo, motivando y retroalimentando con palabras y gestos expresivos, tal y como se expresa en "buena comunicación y actitud motivadora: con tono de voz, implicación con palabras y gestos, refuerza la actividad, recurre al feedback" (1:14); "explicación clara y motivadora, control de la clase pasando por todos los grupos, corregía y daba feedback" (1:43).

También la capacidad para dirigir a los grupos hacia el objetivo, requiere del dominio de las habilidades comunicativas de interactividad, desarrollando un "feeling" relacional con el que hacer fluir un orden fundamentado en el entendimiento mutuo y las relaciones interpersonales, basadas en el diálogo y la complicidad; esto se observa en "buena capacidad de liderazgo, buena conexión con el grupo y "feeling" explicando de forma clara y concisa" 
(1:17); "se comunica con feedback constante, positivo, natural y se relaciona con el alumnado, es parte de ellos, aunque jugando un papel diferente, como dinamizador" (1:31).

\section{DISCUSIÓN Y CONCLUSIÓN}

Para responder sobre las competencias transversales que están presentes en las prácticas formativas de los estudiantes durante el desarrollo de la asignatura Expresión y comunicación corporal, se observan tres competencias especialmente relevantes para los estudiantes participantes en el estudio: liderazgo educativo, competencia comunicativa, y competencia para la planificación y organización docente.

De las dimensiones que configuran el liderazgo educativo, se destacan la motivación, animación y dinamización grupal. Así, la capacidad de motivar se posiciona como una función para conectar con lo mejor de cada persona e impulsarla hacia una versión superior de sí misma y hacia un mayor crecimiento personal (Lorenzo, 2005). Para Vázquez, Bernal y Liesa (2014), la capacidad de motivar, dinamizar, animar, movilizar y potenciar son funciones del liderazgo que contribuyen a la capacidad de orientar y guiar, que en el ámbito educativo se traducen en dirigir, conducir o enseñar el camino al alumnado, a fin de conseguir que logre los propósitos previstos, en este sentido se aprecian coincidencias con Gento y Ruiz (1996) cuando describen que el auténtico líder consigue dinamizar personas y grupos hacia una determinada dirección, moviliza y despierta entusiasmo, anima a hacer salir lo mejor de cada uno, transmite energía y es impulsor de ideas a fin de arrastrar a otros hacia la meta común.

Pero dirigir, conducir y guiar requiere de la capacidad de visión de dirección hacia dónde avanzar, saber hacia dónde dirigirse es saber hacerse una imagen de futuro, que requiere de poner en marcha un plan y unos objetivos, y ponerse en acción para comunicar a su grupo, de forma precisa, la dirección prevista y las tareas necesarias para alcanzar tales metas. En este sentido, se coincide con Fraile (2013) cuando establece para los educadores físicos la necesidad de cara a su ejercicio docente, de establecerse objetivos claros y esclarecer lo prioritario para con las necesidades de su grupo, a fin de planear el futuro conjunto a través de específicas líneas de actuación.

Aparece además como destacada la dimensión prosocial del liderazgo educativo: el líder prosocial decide tomar la iniciativa y asumir la responsabilidad de que las cosas sucedan, a partir de sus valores y convicciones cuidadosamente meditados e internalizados. Por ello, el líder proactivo crea su propio clima emocional, positivo, optimista, creativo, solidario, porque sabe que el contagio emocional depende de él (Goleman, 2014), y los ambientes de aula que se generan bajo su liderazgo resultarán de su estado emocional siendo él mismo el emisor emocional principal y responsable del clima emocional generado. El líder tiene, por tanto, un poder de contagio emocional que afecta a los sentimientos y emociones de su alumnado, a su motivación y deseo de participación y, por ende, a la creación de un ambiente de aula favorecedor de los aprendizajes.

Con relación a la competencia comunicativa se reconocen algunos rasgos paralingüísticos como las cualidades fónicas (claridad, intensidad, volumen y proyección de la voz) que favorecen la transmisión del mensaje didáctico con convicción, para lo cual es necesario evitar la monotonía de la entonación, variar la velocidad del discurso, realizar cambios tonales (emplear tonos agudos y mayor velocidad en la dicción para llamar la atención, o menor velocidad y tonos más graves para enfatizar algo importante del 
mensaje) o variaciones en el volumen. De la misma manera, tal y como describían Ekman y Friesen (1969), se valora la importancia del lenguaje corporal, que si bien se acompaña de gestos durante el proceso de transmisión verbal de la información, también se preocupa de la presencia comunicadora y relacional del cuerpo en el espacio, de forma que el potencial del movimiento corporal acompaña a la palabra y la complementa.

Para Ambady y Roshental (1993) y Prozesky (2000) los buenos docentes evidencian un perfil comunicativo no verbal rico, variado e intenso; son expresivos con su cuerpo, mirada, rostro, manos, voz y cuerpo; permanecen más en pie que sentados y aprovechan su movimiento en el espacio del aula para comunicar desde diferentes puntos de vista y orientados a su audiencia. Igualmente, de acuerdo con Ortiz (2002), se considera necesario en la formación del profesorado de educación física el favorecimiento de situaciones prácticas para experimentar situaciones de uso de los espacios y las distancias en las relaciones interpersonales, no solamente para comunicar mejor, sino para aprovechar el encuentro comunicativo a fin de mejorar las relaciones interpersonales con los demás.

Se aprecia también que comunicación y liderazgo son dos competencias que de forma complementaria facilitan el feedback, de manera que liderar acompañando y guiando necesita del uso de los recursos comunicativos verbales y no verbales, para animar, motivar, corregir y reforzar. Para Alves (2000) un buen nivel de comunicación regula el rendimiento de los grupos y las personas, favoreciendo el entendimiento, el respeto y el ánimo mutuo, la sensación generalizada de satisfacción en el aprendizaje, así como un ambiente amigable proclive al favorecimiento del sentido de pertenencia grupal. De acuerdo con Martens (1987), la asertividad, la empatía, las habilidades comunicativas, la confianza y el aprecio a los demás redundan en la generación de un "feeling" desde el que conectar con el grupo, efecto que es percibido como favorecedor de la complicidad con los miembros del grupo y de su aceptación voluntaria del liderazgo (Lorenzo, 2005).

La planificación de la actividad educativa es un instrumento al servicio de los centros docentes cuya finalidad es dar a conocer sus objetivos y garantizar la coordinación de las actividades propuestas, cumpliendo con los criterios y requisitos propios de una enseñanza eficiente. Se aprecia coherencia en la presencia de los elementos del currículum en la práctica de aula; los objetivos previamente planificados y previstos en las programaciones están presentes y localizables en el contraste de los evaluadores, cuando comparan tales programaciones con las actuaciones docentes. Los objetivos son uno de los componentes curriculares claves en la programación, ya que recogen y conectan las finalidades educativas de la institución y la intencionalidad del profesorado, determinando que sus propósitos y aspiraciones didácticas se reflejen en la práctica (Gimeno-Sacristán, 1988).

En lo referente a la progresión en la implicación motriz y cognitiva de las tareas, se reconoce la necesidad de comenzar con propuestas que faciliten al alumnado su comprensión y ejecución. De acuerdo con Viciana, Salinas y Lozano (2006), se trata de ir alcanzando nuevos retos en función de la dificultad, garantizando un mayor control en los procesos de comprensión y de ejecución motriz, presentando diferentes posibilidades y variantes de actuación, desde distintos niveles de exigencia, con objeto de ir superando las dificultades y problemas que puedan surgir durante la práctica.

Con relación a la elección de los estilos metodológicos, a pesar de que no se aprecia variedad en su elección, ya que los contenidos de expresión corporal determinan el uso prioritario de aquellos estilos que favorezcan la creatividad, la improvisación y la toma de decisiones (Romero-Martín, 2015), sí se reconoce una coherencia entre la selección 
de los métodos activos con el objetivo a alcanzar y la actividad o tarea seleccionada para conseguirlo. La elección de las estrategias de enseñanza tendrá en cuenta los diferentes ritmos de aprendizaje del alumnado, así como su capacidad de aprender por sí mismo (Baena-Extremera et al., 2010), debiendo desarrollarla según las intenciones educativas previstas (López, 2009).

Finalmente, se quiere subrayar que a partir de esta experiencia los estudiantes despliegan ciertas competencias docentes relacionadas con la capacidad de identificar y desarrollar habilidades cognitivas superiores (Sanz de Acedo, 2010), al implicarse desde la práctica de expresión corporal en la resolución de problemas, adaptando los fundamentos teóricos de la asignatura (saberes teóricos sobre la expresión corporal); diseñando sus aplicaciones a través de simulacros docentes (procedimientos y estrategias docentes que faciliten la aplicación de la expresión corporal en determinados contextos educativos); y tomando conciencia tanto del saber ser como del saber estar, desde un trabajo en equipo colaborativo entre estudiantes "docentes" y "observadores-coevaluadores", como parte de un proceso responsable de reflexión sobre sus competencias profesionales para el ejercicio docente y sus niveles de desarrollo alcanzados (Lancaster, Waught \& Wood, 2008).

Cabe subrayar que la internacionalización y la globalización exigen una mayor transparencia en las competencias que adquieren los estudiantes universitarios (Kuhn \& Zlatkin-Troitschanskaia, 2011), y, por consiguiente, exige que se centre el interés formativo en su evaluación. Así, entre una evaluación tradicional centrada en la medición, carente de retroalimentación más allá de la mera calificación sancionadora, y una evaluación formativa y compartida, centrada en el aprendizaje y la retroalimentación para una mejora constante en el desarrollo y adquisición de competencias (Asún, Romero, \& Chivite, 2017), los sistemas de evaluación formativa se vislumbran como los más adecuados para conseguir este objetivo formativo (López-Pastor, 2009).

Por lo que se refiere a las pruebas y el registro de datos, la evaluación formativa hace uso de un continuo y variado sistema de registro de información (textual, audiovisual) y aplican un feedback constante (autoevaluación, heteroevalaución, coevaluación), que favorece mejores resultados académicos y mayores tasas de éxito entre los estudiantes, haciéndoles más conscientes y responsables de lo que aprenden y llevan a la práctica, siendo sus aprendizajes más significativos, aplicativos y eficientes (Carrillo de la Peña et al., 2009; Fraile et al., 2013; Romero et al., 2014).

Tal vez como aspecto a replantear como propuesta de futuro, sería necesario analizar de forma mucho más pormenorizada las imágenes de video captadas, en relación con los comportamientos docentes desarrollados en los simulacros. A través del videoanálisis es posible reconocer e identificar elementos del lenguaje comunicativo no verbal y su relación con el liderazgo docente ejercido, las relaciones interpersonales establecidas y el comportamiento emocional observado. Es posible así dotar de mayor fuerza al sujeto con capacidad narrativa, que poniendo voz e imagen a su experiencia, también la colma de significado, haciendo emerger otras caras del fenómeno objeto de investigación, a través del análisis de los microdetalles registrados mediante video (Baer \& Schnettler, 2014).

En esta experiencia se genera un proceso de interacción y de diálogo entre profesores y estudiantes como parte del procedimiento de evaluación formativa, a través de la heteroevalaución, la coevaluación y la autoevaluación. En definitiva, la exigencia competencial y la retroalimentación que se va desarrollando supone un camino sin retorno y de un óptimo aprendizaje. 


\section{REFERENCIAS BIBLIOGRÁFICAS}

Alves, J. (2000). Liderazgo y clima organizacional. Revista de Psicología del Deporte, 9(1-2), 123 133.

Amado, D., Sánchez-Miguel, P. A., Leo, F. M., Sánchez-Oliva, D., \& García-Calvo, T. (2012). Adaptación a la expresión corporal del cuestionario de Apoyo a las Necesidades Psicológicas Básicas. Electronic Journal of Research in Educational Psychology, 10(27), 867-884.

Ambady, N., \& Rosenthal, R. (1993). Half a minute: predicting teacher evaluations from thins slices of behavior and physical attractiveness. Journal of Personality and Social Psychology, 64(3), 431-441.

ANECA. (2005a). Libro Blanco. Título de Grado en Magisterio. Madrid: Agencia Nacional de la Evaluación de la Calidad y la Acreditación.

ANECA. (2005b). Libro Blanco. Título de Grado en Ciencias de la Actividad Física y el Deporte. Madrid: Agencia Nacional de Evaluación de la Calidad y la Acreditación.

Aparicio, J. L., \& Fraile, A. (2016). Las competencias interpersonales en la formación del profesorado de educación física a través de un programa de expresión corporal. International Journal for 21st Century Education, 10, 21-34.

Asún, S., Romero, M. R., Aparicio, J. L., \& Fraile, A. (2017). Evaluación formativa en la expresión corporal. Tándem, 55, 38-43.

Asún, S., Romero, M. R., \& Chivite, M. (2017). Exploración de sistemas de evaluación formativa entre estudiantes universitarios en la provincia de Huesca. Apunts. Educación Física y Deportes, $127,52-58$.

Baena-Extremera, A., Ruiz, P. J., Granero, A., \& Sánchez, J. A. (2010). Orientaciones en la planificación del currículum en educación física. Espiral. Cuadernos del profesorado, 5(6), 84-90.

Baer, A., \& Schnettler, B. (2014). Hacia una metodología cualitativa audiovisual. El vídeo como instrumento de investigación social. En A. Merlino (Ed.), Investigación cualitativa en Ciencias Sociales. Temas, problemas y aplicaciones (pp. 1-38). Buenos Aires: Cengage Learning.

Carrillo de la Peña, M. T., Bailles, E., Caseras, X., Martinez, A., Ortet, G., \& Perez, J. (2009). Formative Assessment and Academic Achievement in Pre-Graduate Students of Health Sciences. Advances in Health Sciences Education, 14(1), 61-67. doi:10.1007/s10459-007-9086-y

Corominas-Rovira, E. (2001). Competencias genéricas en la formación universitaria. Revista de educación, 325, 299-322.

Ekman, P., \& Friesen, W. (1969). The repertoire of nonverbal behavior: categorie, origins, usage and coding. Semiótica, 1, 49-98.

Fraile, A. (2013). El coaching como estrategia pedagógica para la mejora del autoconocimiento de los educadores deportivos. E-motion. Revista de Educación, Motricidad e Investigación, 1, 1-13.

Fraile, A., \& Aparicio, J. L. (2015). La expresión corporal y el desarrollo de las competencias transversales en la formación del profesorado. Tándem, 47, 1-8.

Fraile, A., López-Pastor, V., Castejón, F. J., \& Romero, M. R. (2013). La evaluación formativa en docencia universitaria y el rendimiento académico del alumnado. Aula Abierta, 41(2), 23-34.

Friese, S. (2012). Qualitative Data Analysis with ATLAS.ti. London: SAGE Publications Ltd.

Gento, S., \& Ruiz, I. (1996). Liderazgo pedagógico del director de una institución educativa de calidad. En A. Villa (Ed.), Dirección Participativa y evaluación de centros (pp. 000-000). Bilbao: ICE-Universidad de Deusto.

Gimeno-Sacristán, J. (1988). El currículum: una reflexión sobre la práctica. Madrid: Morata.

Goleman, D. (2006). Inteligencia Social. La nueva ciencia de las relaciones humanas. Barcelona: Kairós.

Goleman, D. (2014). Liderazgo. El poder de la inteligencia emocional. Barcelona: EdicionesB.

Goleman, D., Boyatzis, R., \& McKee, A. (2002). El líder resonante crea más. El poder de la inteligencia emocional. Barcelona: Plaza \& Janés. 
Estudios Pedagógicos XLIV, $\mathrm{N}^{\circ}$ 2: 39-53, 2018

LA EVALUACIÓN FORMATIVA DE LAS COMPETENCIAS GENÉRICAS EN LA FORMACIÓN DEL PROFESORADO DE EDUCACIÓN FÍSICA

González, J., \& Wagenaar, R. (2003). Tuning Educational Structures in Europe. Bilbao: Universidad de Deusto.

Guba, E. (1985). Criterios de credibilidad en la investigación naturalista. En J. Gimeno-Sacristán y A. Pérez-Gómez (Eds.), La enseñanza: su teoría y su práctica (pp. 148-166). Madrid: Akal.

Knapp, M. L. (1992). La comunicación no verbal. El cuerpo y el entorno. Barcelona: Paidós.

Kuhn, C., \& Zlatkin-Troitschanskaia, O. (2011). Assessment of Competencies Among University Students and Graduates: Analyzing the State of Research and Perspectives. Meinz: Johanes Gutenberg Universiy.

Lancaster, J., Waught, L., \& Wood, J. (2008). Designing and Implementing Student-Centered Assessment. Paper presented at the Annual Assessment Conference, Texas AandM University. http://assessment. tamu.edu/resources/conf_2008/Wood_StudentCenteredAssessment.pdf

Learreta, B., Sierra, M. Á., \& Ruano, K. (2005). Los contenidos de la expresión corporal. Barcelona: INDE.

López, A. (2009). La elaboración de los documentos de planificación: una actividad cuestionada en la formación de maestros. REIFOP, 12(2), 49-57.

López-Pastor, V. (2009). Evaluación formativa y compartida en Educación Superior. Madrid: Narcea.

Lorenzo, M. (2005). El liderazgo en las organizaciones educativas: revisión y perspectivas actuales. Revista Española de Pedagogía, 63(232), 367-388.

Martens, R. (1987). Coaches Guide to Sport Psychology. Champaign. Illinois: Human Kinetics.

Maulini, C., Fraile, A., \& Cano, R. (2015). Competencias y formación universitaria del educador deportivo en Italia. Estudios Pedagógicos, 41(1), 167-182. doi:10.4067/S071807052015000100010

Mehrabian, A. (1971). Silent Messages. Belmont: Wadsworth.

Mehrabian, A. (1972). Nonverbal Communication. Chicago: Aldine-Atherton.

Miles, M., Huberman, M., \& Saldaña, J. (2014). Qualitative Data Analysis: A Methods Sourcebook. London: SAGE Publications.

Monereo, C. (2014). Enseñando a enseñar en la universidad. La formación del profesorado basada en incidentes críticos. Barcelona: Octaedro.

Ortiz, M. M. (2002). Expresión Corporal. Una propuesta didáctica para el profesorado de Educación Física. Granada: Grupo Editorial Universitario.

Poyatos, F. (1994). La comunicación no verbal: cultura, lenguaje y conversación. Madrid: Itsmo.

Preston, P. (2005). Nonverbal Communication: Do you really say what you mean? Journal of Healthcare Management, 50(2), 83-86.

Prozesky, D. R. (2000). Communication and effective teaching. Journal of Community Eye Health, 13(35), 44-45.

Romero-Martín, R. (2015). Expresión Corporal en Educación Física. Zaragoza: Servicio de Publicaciones de la Universidad de Zaragoza.

Romero, M. R., Fraile, A., López, V., \& Castejón, F. J. (2014). Relación entre sistemas de evaluación formativa, rendimiento académico y carga de trabajo del profesor y del alumno en la docencia universitaria. Infancia y Aprendizaje, 37(2), 310-341. doi:10.1080/02103702.2014.918818

Ruano, K. (2013). El despertar de las emociones. Un trabajo corporal. Multiárea. Revista de Didáctica, 6, 311-354.

Salmerón, L. (2013). Actividades que promueven la transferencia de los aprendizajes: una revisión de la literatura. Revista de educación, Volumen extraordinario, 34-53.

Sanz de Acedo, M. L. (2010). Competencias cognitivas en educación superior. Madrid: Narcea.

Schneckerberg, D., \& Wildt, J. (2005). Understanding the concept of ECompetence for Academic Staff. University of Dormunt: Center for research on Higher Education and Faculty Development.

Schön, D. (1992). La formación de profesionales reflexivos. Hacia un nuevo diseño de la enseñanza y del aprendizaje en los profesionales. Barcelona: Paidós.

Tejada, J., \& Navio, A. (2004). Las competencias profesionales (documento mimeografíado). 
Universidad Autónoma de Barcelona, Departamento de Pedagogía Aplicada.

Vázquez, S., Bernal, J. L., \& Liesa, M. (2014). La conceptualización del liderazgo: una aproximación desde la práctica educativa. Revista Electrónica Iberoamericana sobre calidad, eficacia y cambio en la Educación, 12(5), 79-97.

Velázquez, C., Fraile, A., \& López-Pastor, V. (2014). Aprendizaje cooperativo en Educación Física. Movimento: Revista da Escola de Educação Física, 20(1), 239-259.

Viciana, J., Salinas, F., \& Lozano, L. (2006). La planificación de la sesión de Educación Física: tipos de sesiones y ejemplos. Lecturas: Educación Física y Deportes, 97, 1-15.

Villardón-Gallego, L. (2015). Competencias Genéricas en Educación Superior: metodologías específicas para su desarrollo. Madrid: Narcea.

Yániz, C. (2007). Competencias en la universidad. De la utopía a la pragmatopia. DIDAC, 49, 4-9.

Zabala, A., \& Arnau, L. (2007). La enseñanza de las competencias. Aula de innovación educativa, $161,40-46$. 
